\title{
LAS TECNOLOGÍAS DE ENERGÍA RENOVABLE EN LA PRENSA ESCRITA COSTARRICENSE ${ }^{1}$
}

\section{MEDIA COVERAGE REPRESENTATIONS ABOUT RENEWABLE ENERGIES IN COSTA RICAN NEWSPAPERS}

\author{
David Chavarría Camacho*
}

RESUMEN

El presente trabajo analiza la cobertura en prensa escrita sobre el tema de la energía renovable y las Tecnologías de Energía Renovable (TER), asimismo, se analiza cómo dicha información ha sido expuesta en el caso costarricense por los periódicos La Nación y El Financiero, en el periodo comprendido entre enero de 2006 y diciembre de 2011, considerado como el periodo de surgimiento y establecimiento de las discusiones públicas más relevantes con respecto al tema. Se realiza un análisis general de la forma de exposición de la información y del contenido a través de las representaciones sobre el tema, a partir de algunos trabajos surgidos en los últimos años en América Latina y el resto del mundo, relativos a la comunicación de la ciencia y la cobertura periodística en temas de ciencia y tecnología.

PALABRAS CLAVE: COSTA RICA * FUENTE DE ENERGÍA RENOVABLE * COMUNICACIÓN * PRENSA * CIENCIA

\section{ABSTRACT}

This paper analyzes the newspaper's coverage on the subject of renewable energy and renewable energy technologies and how that information has been exhibited in the Costa Rican case by the newspapers La Nacion and El Financiero between January 2006 and December 2011 (considered the period of emergence and establishment of the most important public discussions on the issue). It's presented a general analysis of the form of presentation of information and content through the representations analysis on the subject, taking as a starting point some works that have emerged in recent years in Latin America and the rest of the world, concerning to the communication of science and technology.

KEYWORDS: COSTA RICA * RENEWABLE ENERGIES SOURCES * COMMUNICATION * PRESS * SCIENCE

1 La presente investigación contó con el apoyo del Programa de Investigación Ambiente, Ciencia, Tecnología y Sociedad (ACTS) del Centro de Investigaciones Históricas de América Central (CIHAC). El autor agradece la invaluable guía de la historiadora MSc. Andrea Montero Mora durante la elaboración de este estudio, en la cual se muestran algunos de sus resultados más importantes.

* $\quad$ Escuela de Estudios Generales de la Sede del Atlántico de la Universidad de Costa Rica (UCR). david.chavarriacamacho@ucr.ac.cr 
LA COBERTURA DE LA PRENSA ESCRITA

EN TEMAS DE CIENCIA Y TECNOLOGÍA.

CONSIDERACIONES PARA EL CASO DE AMÉRICA LATINA

En los últimos años, ha aumentado el interés en América Latina en publicar investigaciones que vinculen los temas de la ciencia $y$ tecnología con la forma en la que son expuestos por los medios de comunicación. En dicha región, existen acercamientos aislados que tienen como característica común su dificultad para mantener continuidad en el análisis, considerándose de vital importancia "desarrollar metodologías más sólidas de análisis y evaluación de la cobertura de la prensa con respecto a temas de ciencia y tecnología" (Massarani y Buys, 2008, p.116). Según estos autores, la importancia de analizar la cobertura periodística radica en el hecho de que son fuentes con un rol importante en lo que respecta a la "comprensión, el juicio de valor y las actitudes del público en general sobre temas relacionados con ciencia $y$ tecnología [ya que] son una fuente importante de información consultada por el público en general y pueden influir en los preconceptos" que se tienen sobre temas científicos específicos (Sarudiansky, 2016, p.26).

Bruno Takahashi y Mark Meisner (2012), desde el enfoque de la comprensión pública de la ciencia (Public Understanding of Science), se han preocupado por estudiar las representaciones mediáticas del cambio climático en la prensa peruana, con lo cual demuestran cómo las voces externas en la prensa escrita local opacan la cobertura mediática sobre las estrategias de estos países para mitigar los efectos negativos de dicho fenómeno atmosférico. De igual forma, el historiador Ronny Viales, quien analiza este mismo problema para el caso de Costa Rica, ha argumentado que a través de los medios escritos de este país "se reproduce la información que generan las grandes agencias noticiosas de los países ricos/desarrollados, pero los que ya han tomado posición sobre la tendencia dominante en la geopolítica mundial del cambio climático y del calentamiento global" (Viales, 2012, sp.).

Desde esta perspectiva, el artículo de Luisa Massarani y Bruno Buys titulado "Cuando la ciencia es noticia: una evaluación de la sección de ciencia en nueve países de América Latina", que pertenece a un ambicioso trabajo compilatorio regional acerca de la comunicación de la ciencia (Lozano y Sánchez, 2008), realiza un intento por responder en qué medida la ciencia nacional es presentada en los periódicos, considerando el hecho de que se encuentra mediatizada por las discusiones que se llevan a cabo en los países desarrollados. Estos autores encuentran diferencias considerables en lo que respecta a la valoración de la ciencia nacional en cada país. Se destaca el caso de Argentina por el gran valor que la prensa le da a la ciencia y la tecnología local. Además, su estudio señala el hecho de que Costa Rica presenta una cantidad considerable de notas periodísticas ${ }^{2}$, a pesar de contar con un sistema institucional de ciencia y tecnología, $y$ una práctica de periodismo científico menos consolidados que países como Brasil, Argentina y México. Como explicación a este fenómeno, señalan que Costa Rica "tiene un movimiento reciente hacia el periodismo científico, incluso con la creación de una asociación nacional" (p.126), la Asociación de Periodismo Científico Costarricense (APCC), que es patrocinada por el Consejo Nacional para Investigaciones Científicas y Tecnológicas (CONICIT).

En estas investigaciones son menos evidentes los análisis de la información relativa a la implementación de nuevas tecnologías en general y de la generación de energías renovables en específico, en los que se privilegian los temas relativos a las ciencias de la salud. Por el contrario, en el presente artículo se analiza la cobertura en prensa escrita sobre el tema de la energía renovable y las tecnologías de energía renovable (TER), en el caso específico de Costa Rica, por los periódicos La Nación y El Financiero, durante el periodo comprendido entre enero de 2006 y diciembre de $2011^{3}$.

$2 \quad$ Los autores contabilizan únicamente el periódico La Nación.

3 El periodo analizado en esta investigación cubre una de las etapas más importantes en la historia de la energía renovable en Costa Rica. Su marco temporal responde a que se firma el Protocolo de Kyoto sobre el cambio climático en el año 1997, 


\section{METODOLOGÍA PARA EL ANÁLISIS DE LA INFORMACIÓN}

Se realizó un análisis de la información referente a las energías renovables en los periódicos La Nación y El Financiero. Previamente se recolectaron y sistematizaron mediante la base de datos digital de ambas empresas, un total de 398 noticias entre enero de 2006 $y$ diciembre de 2011, que contienen la frase "energía(s) renovable(s)" ${ }^{4}$. Para sistematizar e interpretar de manera más ordenada la información, se emplearon cinco categorías de análisis: origen de la nota (nacional o internacional), agencia o empresa emisora, problemática tratada (económica, climática y/o de contenido ambiental), temas principales (economía/mercado, industrial/empresarial, política nacional, público/sociedad civil, relaciones internacionales, transferencia de tecnología y/o recursos) y tipo de actor (públicos, privados, internacionales, individuales, colectivos) (tabla 1).

\section{TABLA 1 \\ PERIÓDICOS LA NACIÓN Y EL FINANCIERO \\ INDICADORES ESPECÍFICOS PARA EL ESTUDIO DEL ORIGEN DE LOS ARTÍCULOS REFERIDOS A ENERGÍAS RENOVABLES EN AMBOS PERIÓDICOS}

2006-2011

VARIABLES

CONTENIDO

Origen

Internacional Emitido en Costa Rica o por un corresponsal del medio en algún país de Nacional Centroamérica.

Agencia

Emisora de la nota periodística. Se sistematiza solo para las noticias de origen internacional.
Problemática
Señala (cuando existe) la principal problemática tratada por el artículo.
Económica
Trata asuntos estrictamente económicos, como variaciones en el precio
internacional del petróleo y problemas en el financiamiento de las TER.
Climática
Señala como problemática el Cambio Climático y/o el Calentamiento Global.
Ambas
Cuando refiere a la combinación de problemas económicos y climáticos.
Contenido Ambiental
Relativo a conflictos de contenido ambiental.

Continúa...

generado a partir de la Convención en el Marco de las Naciones Unidas sobre el Cambio Climático (CMNUCC), llevado a cabo en 1992. Sin embargo, este no entra en vigencia sino hasta el 2005, por lo que a partir de este momento, las tecnologías renovables comienzan a formar parte importante de las políticas nacionales $y$ regionales referentes a la generación, ahorro y distribución energética. Igualmente, una publicación del Banco Mundial del 18 de setiembre de 2006, marca este punto de partida, ya que señala una serie de obligaciones internacionales referentes a la transferencia de tecnología hacia los "países en desarrollo" con el fin de combatir el cambio climático y la pobreza. También establece las bases para la inversión y financiamiento de las energías renovables.

4 Esta búsqueda se realizó a través del sitio web de ambos periódicos. La Nación (http://www.nacion. com/buscador/?text=Buscar...) y El Financiero (http://www.elfinancierocr.com/buscador/?text=). 


\section{VARIABLES}

Temas principales

Economía/Mercado

Industrial/Empresarial

Política Nacional

Público/Sociedad Civil

Relaciones Internacionales

Transferencias de tecnología
CONTENIDO

Cada artículo consultado puede tratar varios temas, todos los temas o ninguno de ellos.

Economía tecnológicamente dirigida, competitividad/actividades de exportación, apertura de mercados.

Intereses económicos y políticas industriales o empresariales, clústers en TER, innovación industrial/empresarial, financiamiento de negocios.

Discusiones sobre políticas del Estado en materia de regulación, impuestos, incentivos, gobernabilidad, I+D público, sobre materia referente a TER.

Presión/manifestaciones públicas en materia de generación eléctrica, opinión pública, actores locales y ciudadanía en general.

Relaciones Norte-Norte, Sur-Sur, Norte-Sur, expedición de documentación internacional, discusiones globales/regionales, organismos internacionales $y$ conflictos internacionales relativos a TER.

Internacional: transferencia tecnológica, relaciones de dependencia económica, inversión de un Estado en otro, en lo que respecta a TER.

Tipo de actor

Públicos

Instituciones, empresas u organismos que responden a los intereses del Estado (ejemplo: ARESEP, Asamblea Legislativa, ICE).

Privados Instituciones, empresas u organizaciones que responden a intereses privados (ejemplo: uccaEP, Tío Pelón, Google).

Internacionales Instituciones, empresas u organismos que trascienden el ámbito de lo nacional (ejemplo: Unión Europea, Banco Mundial, G-20).

Individuales

Individuos que se destacan del colectivo y no muestran vínculo directo con alguno de los otros actores referidos (ejemplo: Al Gore, Paul David Hewson "Bono", Alejandro Sánz).

Colectivos

Conjunto de individuos (ejemplo: campesinos, público lector, vecinos, comunidad).

Fuente: Elaboración propia a partir de la recolección y sistematización de noticias del periódico La Nación y El Financiero, 2006-2011.

Se incluyó una última categoría que corresponde al Tipo de Fuente de Energía, en donde se tomó en cuenta la energía generada a partir del Carbón, Gas Natural, Petróleo, Propano, Uranio, Biomasa, Geotérmica, Undimotriz, Hidroeléctrica, Solar, Eólica, Hidrógeno y Otras. A las tres primeras en conjunto se les denominó como hidrocarburos y para efectos de este estudio son - junto con el propano $y$ el uranio- las llamadas fuentes de energía no renovables. Las restantes se consideran fuentes de energía renovables. 


\section{RESULTADOS PRINCIPALES}

A partir del análisis numérico acerca del origen nacional o internacional de las noticias, se muestra solamente el gráfico correspondiente al caso de La Nación, debido a que el 100\% de las noticias de El Financiero recopiladas son de carácter nacional. Este último medio, hablando específicamente de las noticias referentes a las
TER, emite en su mayoría, información especializada que trata temas relativos al contexto económico y empresarial de la producción de energía a nivel nacional y centroamericano. Según la clasificación llevada a cabo, las noticias de El Financiero que poseen un país de origen distinto a Costa Rica, fueron redactadas en su totalidad por corresponsales de la misma empresa, localizados en algún país centroamericano.

GRÁFICO 1

\section{LA NACIÓN: VARIACIÓN EN LA CANTIDAD DE ARTÍCULOS SEGÚN ORIGEN NACIONAL \\ O INTERNACIONAL 2006-2011}

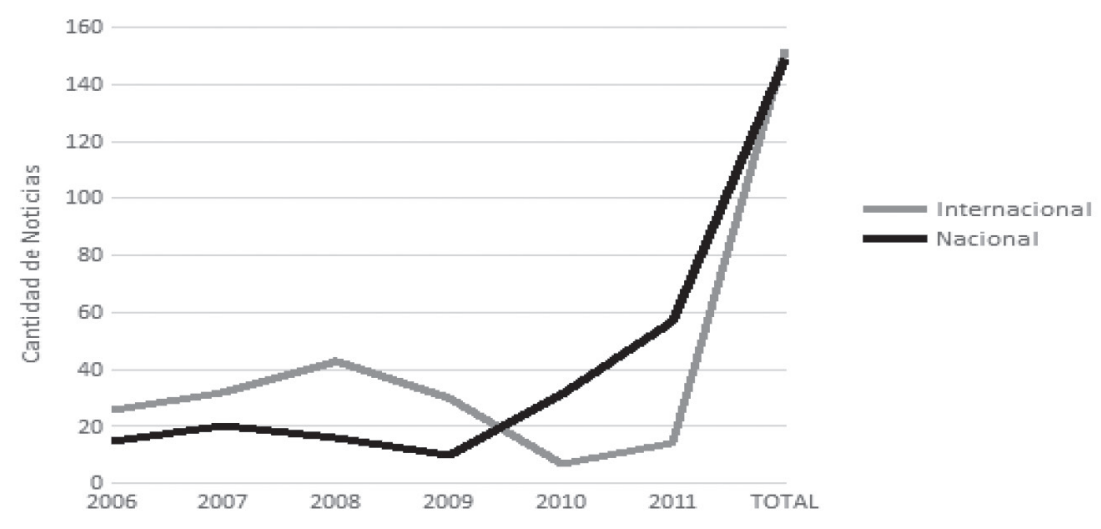

Fuente: Elaboración propia a partir de la recolección y sistematización de noticias del periódico La Nación, 2006-2011.

El Gráfico 1 muestra para el caso de La Nación, la variación en la cantidad de artículos según su origen, en el cual las noticias nacionales tienen una tendencia creciente. Entre 2006 y 2009 se mantiene relativamente estable, pero a partir de ese año comienza a crecer de forma acelerada. Caso contrario a las notas de origen internacional, que a nivel general presenta una tendencia sostenida hacia el decrecimiento, principalmente a partir del año 2008.

Se puede argumentar, entonces, que a pesar de que las noticias referentes a las energías renovables disminuyen en cantidad, existe una mayor disposición a publicar información sobre este tema en el caso nacional. 


\section{GRÁFICO 2 \\ PERIÓDICOS LA NACIÓN Y EL FINANCIERO: SECCIONES CON MAYOR CANTIDAD DE NOTICIAS REFERENTES A LA ENERGÍA RENOVABLE}

(2006-2011)
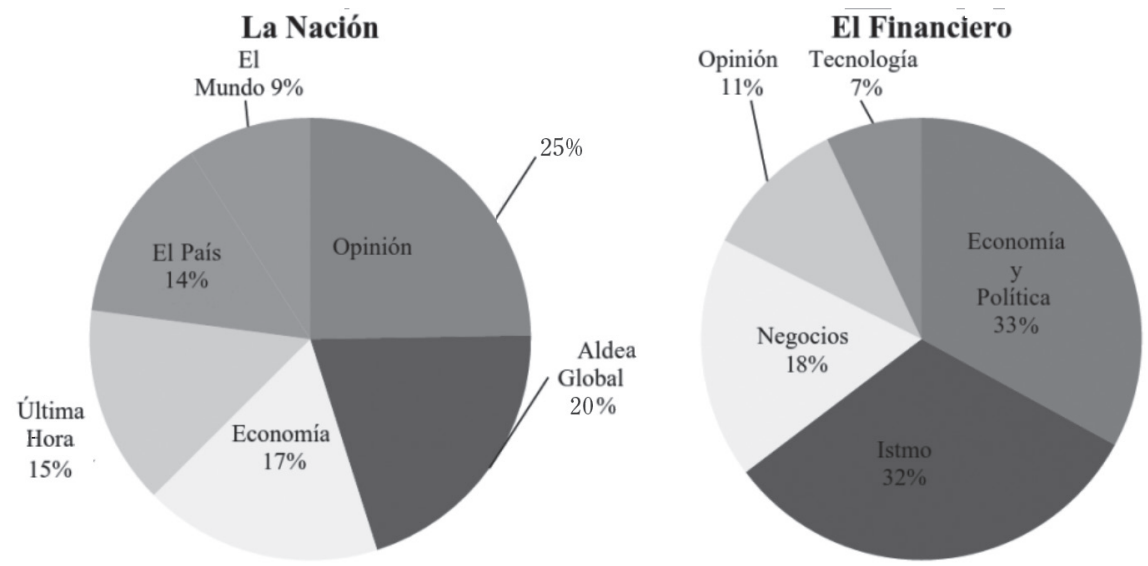

Fuente: Elaboración propia a partir de la recolección y sistematización de noticias del periódico La Nación y El Financiero, 2006-2011.

El gráfico 2 muestra las secciones donde se concentra la mayor cantidad de noticias en ambos medios. Para el caso de La Nación, la mayor parte se encuentran en la sección Opinión. Según el descriptor del medio, esta sección expone "la posición oficial del diario, por medio del editorial, $y$ una amplia variedad de puntos de vista sobre los hechos más relevantes nacional e internacionalmente". Estos puntos de vista no necesariamente representan la opinión del medio, sin embargo, se observó también que algunos expertos en el tema energético tienen una cantidad considerable de notas de opinión (Estudio General de Medios, $2011)^{5}$. Esta sección constituye un espacio que poseen diversos actores involucrados en algún asunto de interés nacional y en varios casos plantea verdaderas discusiones sobre el tema, al promover espacios de derecho a la respuesta.

Igualmente, la totalidad de noticias de esta sección trata discusiones en el ámbito público nacional, referidas a alguna problemática

$5 \quad$ Estudio General de Medios, IPsos-Media CR, Descripción general de La Nación, octubre 2011 a setiembre 2012b. Tomado de http://www.nacionmediakit.com/contenido/la-naci\%C3\%B3n.html que puede afectar a la sociedad costarricense en su conjunto. Durante los tres primeros años, incrementa en un 10\%, 19\% y $22 \%$ el total de las noticias. Este aumento entre 2006 y 2008 es importante porque se relaciona directamente con un incremento en los espacios de opinión pública a través de este medio. Para el 2011, la sección llegará a su máximo histórico de $35 \%$, concentrando allí la mayor cantidad de noticias por sección para todo el periodo en estudio. De esta manera, a pesar de que en este análisis no se cuantificó la cantidad de comentarios hechos por los lectores vía sitio web, en esta sección es donde se concentra la mayor parte de estos.

En diversas ocasiones, estos artículos son publicados como respuesta a una crítica directa de otro autor, lo cual genera controversias entre actores involucrados directamente con el desarrollo y la implementación de tecnologías de energía renovable en Costa Rica, comparándolas generalmente con otros países centroamericanos.

Se observan varios actores clave que interactúan en esta sección. Del total de 68 artículos 
procesados, 7 son emitidos por Ricardo Trujillo Molina, quien está a favor de la exploración y el aprovechamiento del gas natural como fuente de energía no renovable; 6 son redactados por la Editorial del diario; 5 por René Castro Salazar, en su condición de Ministro y exministro de Ambiente y Energía (según el año analizado) y 3 de Teófilo de la Torre, como Ministro de Ambiente y Energía durante el primer tercio de la administración Chinchilla, pasando posteriormente a ocupar la presidencia ejecutiva del Instituto Costarricense de Electricidad (ICE). El "éxito" final demostrado por la sección Opinión, se genera a partir de un aumento gradual en la importancia de esta $y$ de la inserción sistemática de discusiones en el ámbito público nacional, tal como señalan los porcentajes anteriormente expuestos.

Para el caso del periódico El Financiero, el gráfico 2 demuestra una concentración importante del $32 \%$ en la sección Istmo. En párrafos anteriores se constata el carácter nacional de las noticias de este medio, y por lo tanto, se observa una concentración en las noticias que retratan los progresos de los países centroamericanos en cuanto a la implementación de TER y de energías renovables en general. Esta sección, según la información emitida por el medio, retrata "los hechos de negocios más importantes en Centroamérica y República Dominicana" (Estudio General de Medios, 2011) ${ }^{6}$. Ante tal situación, surge la pregunta relativa al por qué existe un particular interés por las noticias referentes a TER en estos países.

En primera instancia, cabe aclarar el carácter regional de esta agencia, puesto que su contenido está dirigido al establecimiento de vínculos empresariales entre Costa Rica y los demás países del istmo. Así, el mercado a nivel regional se observa de forma diferenciada según la preponderancia que posee el capital privado en materia energética. Se puede corroborar de esta forma, que los inversores privados (incluyendo a los de capital costarricense) ingresan con mucha más dificultad

6 Estudio General de Medios, octubre 2011 a setiembre 2012 . en el mercado de la generación eléctrica en Costa Rica que en el resto de países centroamericanos. Este tema se ampliará en la sección de discusión.

Del análisis cualitativo de los datos, se puede observar una tendencia a comparar el "progreso" de estos países, con un relativo atraso costarricense en lo que respecta a nuevas TER. Se puede decir que la mayoría de los avances en materia de TER en estos países del istmo, surge por iniciativas privadas y en muchos casos transnacionales. Situación que refuerza el conflicto existente entre productores de energía privados y los estatales en el caso de Costa Rica, lo cual provoca muchas veces la deslegitimación del modelo solidario del ICE. En este sentido, la mayor concentración de noticias de El Financiero se localiza en la sección "Economía y Política" (Estudio General de Medios, 2011) ${ }^{7}$, en la cual se tratan esas controversias para el caso específico costarricense $e^{8}$.

$7 \quad$ La sección Economía y Política de El Financiero es una "sección de análisis que le explica al lector la forma en que las decisiones o acciones en materia económica afectan al sector productivo. Incorpora las voces de los empresarios y de estudiosos de los temas. [El apartado] Legales incorpora a los expertos externos con los periodistas costarricenses para la interpretación de hechos jurídicos que afectan los negocios y la vida económica nacional [y] Columna Tributaria aconsejarán [sic] derecho tributario, gobierno corporativo, propiedad intelectual, derecho bursátil, derecho ambiental derecho laboral" (Estudio General de Medios, octubre 2011 a setiembre 2012).

8 Un 54\% de los artículos de Economía y Política de El Financiero son de escala espacial Nacional. 


\section{GRÁFICO 3 \\ PERIÓDICOS LA NACIÓN Y EL FINANCIERO \\ CONCENTRACIÓN DE NOTICIAS POR ESCALA ESPACIAL* \\ 2006-2011 \\ (PORCENTAJES)}

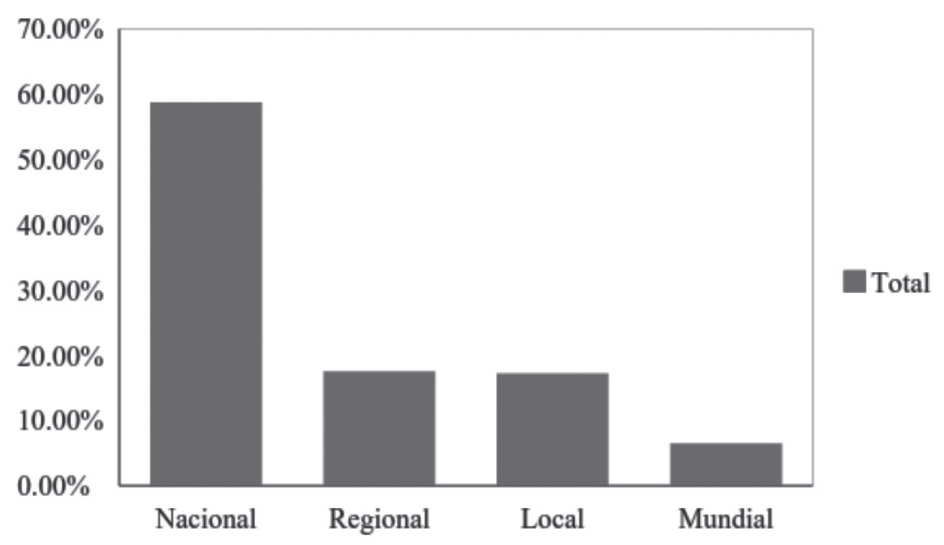

Fuente: Elaboración propia a partir de la recolección y sistematización de noticias del periódico La Nación y El Financiero, 2006-2011.

* El total de artículos mostrado en este gráfico representa una muestra del $77 \%$ del total de las noticias, correspondientes a los cuatro índices datados en este gráfico.

Al observar una muestra del $77 \%$ de las noticias, porcentaje correspondiente a las mayores concentraciones por Escala Espacial tratada y analizar el resumen de datos presentado en el gráfico 3, se notó que la mayor parte de las noticias abordan temas nacionales, independientemente del país que se mencione en el texto. En segundo orden, en las noticias correspondientes a la escala regional se desarrollan temas relativos a las relaciones entre países pertenecientes a una región específica, por ejemplo, los países miembros de la Unión Europea y la región centroamericana.

En cuanto al análisis de la variación a través del tiempo, se extraen algunas conclusiones un tanto más específicas. Del gráfico 4 se puede establecer un comportamiento creciente, tanto de las noticias que analizan la escala Nacional, como de las que analizan el ámbito Local ${ }^{9}$. La conclusión que se establece

9 Como se indicó en el primer apartado de este capítulo, el ámbito local correspondía a un espacio específico dentro de un país. es que a través del periodo en estudio, las noticias tienden a trasladarse del ámbito internacional y regional al ámbito nacional y local.

El punto anterior es importante para constatar el argumento presentado a lo largo de este apartado, en el cual las noticias emitidas por ambos medios, tienden a concentrarse en los asuntos nacionales y deja de lado los internacionales, dominantes en el periodo comprendido entre 2006 y 2008. En este sentido, se traza un primer "periodo" caracterizado como una etapa incipiente en lo que respecta a las noticias referentes a energías renovables en el ámbito nacional, considerando que el número de noticias tomadas de otras agencias significa, en cierto grado, poco interés local por el tema. El segundo periodo comprende los últimos tres años 2009-2011, etapa caracterizada por una inserción más efectiva de los discursos nacionales y regionales referentes a este tipo de fuentes de energía. 


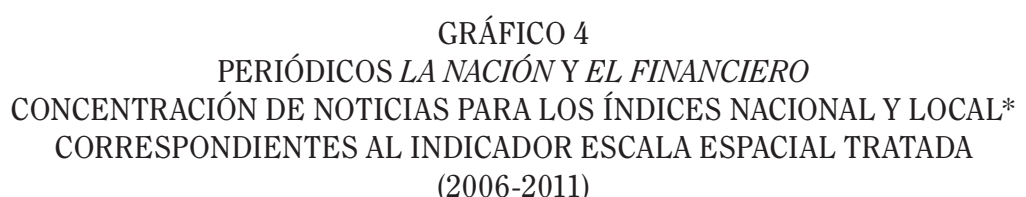

(2006-2011)

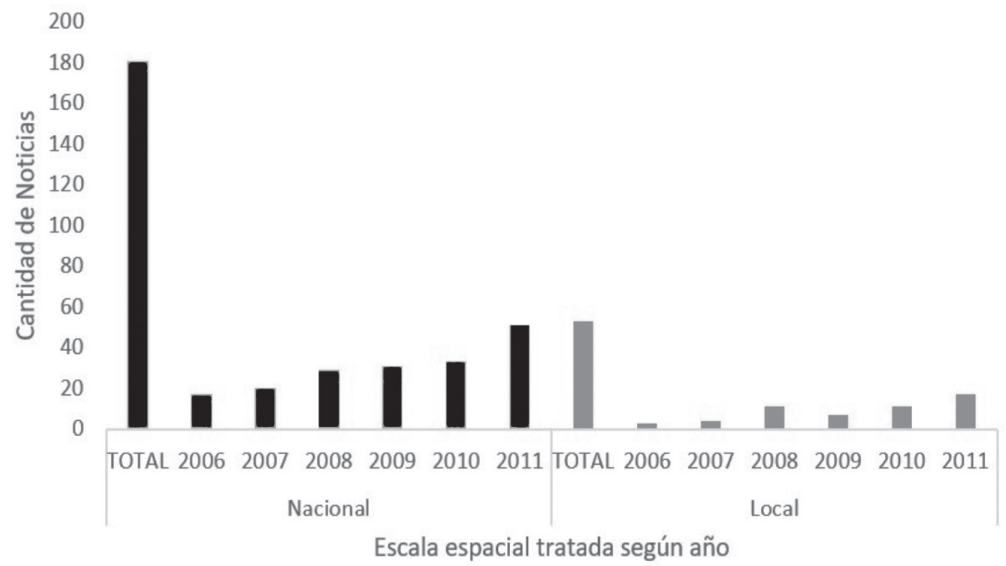

Fuente: Elaboración propia a partir de la recolección y sistematización de noticias del periódico La Nación y El Financiero, 2006-2011.

* El Total de artículos mostrado en este gráfico corresponde a una muestra del $76 \%$ del total sistematizado, porcentaje correspondiente a las mayores concentraciones de noticias por escala espacial tratada.

El análisis de la agencia emisora de la información confirma lo que se ha mencionado, un decrecimiento de las noticias redactadas por empresas internacionales. En el gráfico 5 se observa que la pendiente de la curva se incrementa a partir de 2010 en lo que respecta a las noticias emitidas propiamente por los periódicos, a pesar de que en ese año específico la cantidad total de noticias sobre energías renovables decrece en el caso de La Nación. En este medio de comunicación, la cantidad de noticias redactadas se mantiene relativamente constante du- rante el primer periodo analizado y presenta un repunte a partir del año 2009, que se extiende hasta el 2011. Este comportamiento evidencia que la información generada por las agencias internacionales pierde presencia en forma gradual dentro de los medios nacionales. Paralelamente al hecho de que el interés con respecto al tema de la generación eléctrica transita de lo internacional a lo nacional y comienza a concentrarse en la exposición de las controversias económicas y políticas surgidas entre actores relevantes a nivel regional y nacional. 


\section{GRÁFICO 5 \\ PERIÓDICOS LA NACIÓN Y EL FINANCIERO \\ TENDENCIA DE LAS NOTICIAS REDACTADAS POR AMBAS AGENCIAS \\ 2006-2011}

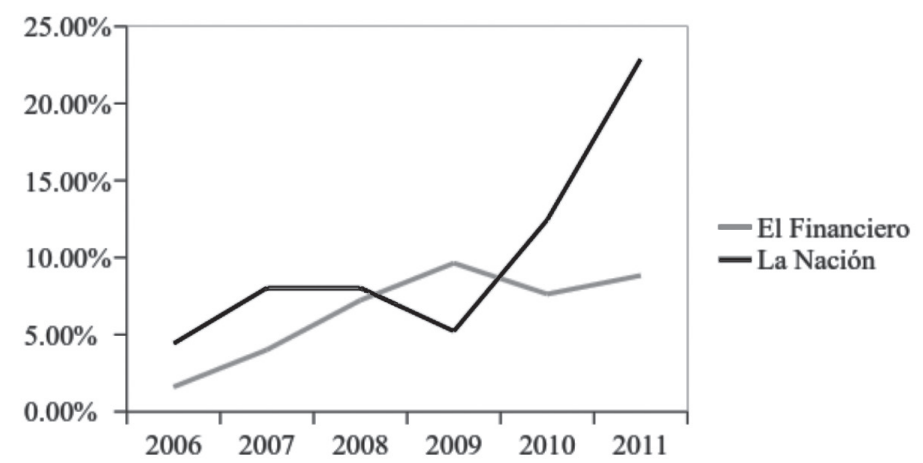

Fuente: Elaboración propia a partir de la recolección y sistematización de noticias de los periódicos La Nación y El Financiero, 2006-2011.

* $\quad$ Este gráfico excluye las noticias tomadas de las agencias internacionales.

\section{DISCUSIÓN}

La categoría de la Problemática tratada por las noticias, se construyó a partir de la lectura detallada de las noticias, con lo cual se pudo constatar la existencia de dos problemas clave expuestos por los medios. En primera instancia, se observa una diversidad de problemas estrictamente económicos, que giran en torno a los cuestionamientos sobre la dependencia al consumo de hidrocarburos. A raíz de ello, se generan inconvenientes más localizados de tipo industrial y empresarial, en lo que respecta a inversión, acceso al crédito y mercados para implementar las TER. Para el caso de Costa Rica, se observa que la principal problemática expuesta consiste en el abasto energético por parte del ICE, que involucra una serie de actores que se enfrentan en el ámbito económico y esto trasciende al ámbito público a través de la prensa.

La segunda instancia es la cuestión climática, que si bien está estrechamente relacionada con el aspecto económico, no se limita a este. Como principal argumento se sostienen los daños de tipo ambiental que ocasiona la emisión de Gases de Efecto Invernadero (GEI), principalmente el Dióxido de Carbono $\left(\mathrm{CO}_{2}\right)$ y el Metano $\left(\mathrm{CH}_{4}\right)$. Igualmente, este argumento genera controversias específicas de tipo políticas y económicas, tanto a nivel nacional como internacional. 


\section{GRÁFICO 6}

PERIÓDICOS LA NACIÓN Y EL FINANCIERO DISTRIBUCIÓN DEL TOTAL DE LOS ARTÍCULOS DE PERIÓDICO PROCESADOS SEGÚN TIPO DE PROBLEMÁTICA PLANTEADA POR SU AUTOR 2006-2011

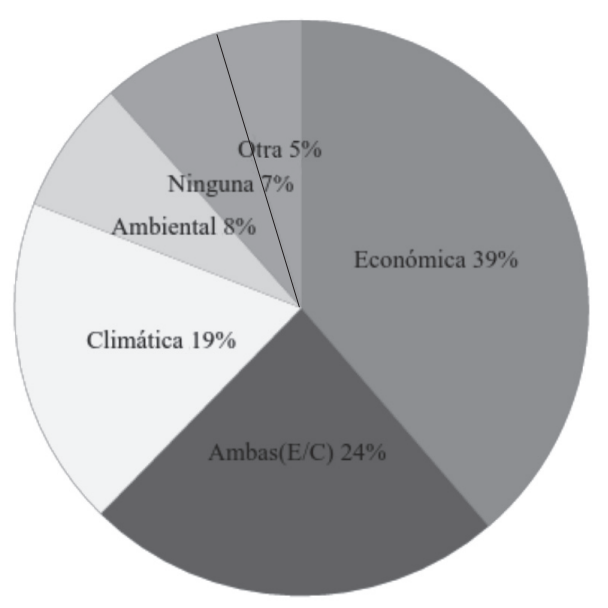

Fuente: Elaboración propia a partir de la recolección y sistematización de noticias del periódico $L a$ Nación y El Financiero, 2006-2011.

El Gráfico 6 muestra la distribución total de las noticias según problemática enfatizada por el autor del texto. Una gran parte de las noticias se ubican en la categoría económica; la de tipo climática ocupa la tercera posición con un $18 \%$ del total ${ }^{10}$. Por otro lado, para observar la estrecha relación entre dichos problemas que existe en el discurso de la prensa, se empleó el indicador Ambas (e/c), en donde se concentra

10 Se debe aclarar que este indicador se refiere estrictamente a menciones directas sobre el cambio climático y el calentamiento global. Esto debido a que muchas de las notas se refieren a la emisión de GEI pero no hacen referencia directa sobre el problema climático, sino desde un enfoque más de tipo industrial y empresarial. Sobre este aspecto no existen estudios que determinen si los discursos que mencionan los GEI están ligados siempre al problema del cambio climático o a la hipótesis del calentamiento global, por lo que se considera que el clasificar estas noticias como problemas de tipo climático no sería el procedimiento más adecuado. casi un cuarto del total de noticias procesadas. Este hace referencia a las notas que mencionan la problemática climática como justificación para generar una respuesta de tipo económica. En cuarto lugar, con un $7 \%$ se identificaron conflictos de tipo ambiental generados a partir de la implementación de TER, caracterizados por mostrar tensiones entre dos o más actores en defensa de un bienestar de tipo material y no directamente por la defensa del medio ambiente (Folchi, 2001).

El principal problema latente que se observa a través del periodo de estudio, es una pugna entre el ICE $y$ los inversores privados por la generación de energía eléctrica. Las empresas privadas vislumbran un lucrativo mercado en la generación eléctrica, por lo que desde la década de los 90 han venido ejerciendo presión política para que se estimule la privatización del sector. En 2000, esta situación se visibilizó plenamente con el "Combo del IcE", donde se movilizó un amplio sector de la sociedad civil contra la privatización de dicha institución.

El ice se caracterizó durante la segunda mitad del siglo xx, porque su oferta de energía primaria se conformó "esencialmente por los hidrocarburos importados y por los recursos hidráulicos y biomásicos" (Blanco y Fajardo, 2005). En tal patrón dual de generación, los hidrocarburos importados suplieron un 70\% de la energía para el año 2004, principalmente para satisfacer la demanda del sector de transportes y la generación eléctrica, lo cual señala la fuerte dependencia hacia dicha fuente. Por otro lado, las cifras sobre la generación eléctrica con fuentes renovables han variado constantemente. Por ejemplo, ese mismo año se caracterizó porque la generación a partir de energía renovable fue de un $98 \%$, mayoritariamente dada por los sistemas de generación hidroeléctrica desarrollados por el Ice.

Sin embargo, para los años correspondientes a este estudio (2006-2011), se da un incremento sustancial en la utilización de plantas de generación térmica propiedad de esta empresa, que funcionan a partir de la quema de diésel y bunker. Esta se constituye en la fuente principal para suplir la demanda cuando las condiciones ambientales no permiten producir 
una cantidad suficiente de energía para cumplir con las demandas del mercado (Blanco y Fajardo, 2005).

Por tal motivo, desde 1990 se decretó la Ley nro. 7200, que regula las relaciones de compra de energía al sector privado, concediéndole capacidad al ICE de adquirir de las empresas privadas hasta un $15 \%$ de la capacidad eléctrica nacional ${ }^{11}$. Los empresarios, a través de un proceso de licitación obtienen tal derecho, para que el ICE, como único distribuidor legal a nivel nacional, lo haga llegar a sus consumidores, obedeciendo a la lógica del modelo solidario (Blanco y Fajardo, 2005).

Sin embargo, la ley sufrió una modificación posterior (nro. 7508, de 1995), incluyendo ahora la posibilidad de que los inversionistas privados inviertan en la construcción de sistemas tecnológicos para la generación privada, que se denominaron "centrales eléctricas de capacidad limitada". Posteriormente, estos bienes se transfieren al ICE a través del mecanismo denominado вот (por sus siglas en inglés, BuildOperate-Transfer, Construir-Operar-Transferir) (Procuraduría General de la República, 1995). De esta forma, diversos "grupos hegemónicos del país (y de toda Centroamérica), ya están tomando decisiones radicales en este campo [en la generación a partir de renovables], movidos por fuertes intereses corporativos que avizoran un nuevo y lucrativo ámbito de negocios" (Cerdas, 2007, p.113).

La principal controversia expuesta por las fuentes periodísticas consultadas, radica en que la generación a partir de fuentes renovables aún no es capaz de suplir energía durante todos los meses del año, debido a que las plantas hidroeléctricas nacionales disminuyen su producción durante la época seca por la reducción del caudal de los ríos (de la Torre, 28 de mayo de 2007). Para solucionar esto, el ICE se vale de la

11 En la Asamblea Legislativa se creó una comisión para discutir sobre el proyecto denominado Ley General de Energía, el cual posteriormente sufre una modificación por una más simple, denominada Ley de Contingencia Eléctrica. En esta comisión, uno de los puntos principales de discusión es la ampliación de este porcentaje de generación por parte de los inversionistas privados. quema de hidrocarburos, así como de una cogeneración al costo para suplir la demanda.

La quema de búnker en las plantas térmicas del ICE, es utilizado como discurso por estos grupos de poder para generar controversias a través de la prensa escrita, argumentando que el modelo de generación eléctrica nacional es deficiente, ya que por un lado no puede suplir de manera adecuada a toda la población y esto genera "apagones" durante algunos meses del año, los cuales, - -hipotéticamente-, iban a ser críticos a partir de 2014, alarmando a los lectores y evidenciando la necesidad de otorgar beneficios a los inversionistas privados, como lo únicos capaces de solucionar el problema (de la Torre, 28 de mayo de 2007).

En este contexto, la editorial del periódico La Nación considera que se debe eliminar el tope de producción privada de $15 \%$, debido a que estas empresas deben proveer al menos el $20 \%$ de la energía eléctrica del país, con el fin de subsanar la deficiencia institucional. Inclusive se pone en discusión la apertura total de la generación nacional (La Nación, 19 de diciembre de 2009). De manera contundente, La Nación apoya la generación privada y señala como "monopólica" la legislación que protege al ICE en materia de compra y distribución de energía (La Nación, 12 de agosto de 2011).

La no apertura, según el medio escrito, incentiva la dependencia a los hidrocarburos cuyos precios tienden al incremento, decayendo paralelamente la economía y perjudicando el medio ambiente por la emisión de GEI (Blanco y Fajardo, 2005). En este sentido, se observa que La Nación ataca indirectamente a algunos sectores de la sociedad civil, al etiquetarlos de tener una "actitud superficial amigable con la naturaleza", por el hecho de que se oponen a la explotación de energía geotérmica en las áreas de conservación del país. Tal comportamiento marcadamente ecologista ocasiona, según este periódico, que continúe la quema desmedida de hidrocarburos.

Este medio apoya la explotación de energía en zonas protegidas e igualmente plantea una crítica fuerte a los grupos comunales que luchan contra la construcción de proyectos de energía renovable en sus localidades. En contraposición 
a las protestas, los medios difunden temas relativos a los "desastres ambientales" ocasionados por los proyectos de generación a partir de hidrocarburos. Igualmente, El Financiero le da especial relevancia a una serie de problemas generados por la quema de hidrocarburos, su almacenamiento $y$ transporte en diversas regiones del país (Castro, 11 de setiembre de 2011) y se expresa a favor de la transición hacia el uso de las energías renovables, acusando al Poder Legislativo de no crear el marco legal para que este tipo de energía pueda ser explotado.

Por otro lado, a pesar de que en Costa Rica se da una sucesión consecutiva de poder por parte del Partido Liberación Nacional (PLN) durante los dos periodos políticos estudiados, existen contradicciones en su proyecto energético, que se hacen evidentes a partir del análisis de los discursos en la prensa. Se observa que Óscar Arias, anterior presidente de la República, llegó a caracterizar de inoperante al gobierno de Laura Chinchilla, al no lograr la aprobación de la Ley General de Electricidad (que contiene 139 artículos), siendo sustituida por una de emergencia que consta de 17 artículos. Arias argumenta que el gobierno de Chinchilla tiene "falta de claridad mental" (Murillo, 11 de agosto de 2011) ${ }^{12}$. Además, genera un discurso a favor de los generadores privados, señalando que estos tuvieron que

12 La Ley de Electricidad 17 812, simplificación del proyecto de Arias, fue propuesta durante el gobierno de Chinchilla y se propone modificar la Ley 7200 de 1990, que autoriza la generación eléctrica autónoma de capital privado, para que el porcentaje de capacidad de venta pase del 15\% al 25\%, y de la misma forma, aumente la producción máxima de cada sistema tecnológico de 20 mw a 30 Mw. La represa hidroeléctrica de Cachí inaugurada en 1966 , produce por ejemplo, una cantidad de $64 \mathrm{MW}$; la nueva planta hidroeléctrica de Diquís tendría la capacidad de generar 660 mw. Además, todos los sistemas de generación privada, debido a esta ley, poseen una capacidad menor de generación que los proyectos de larga escala propiedad del ICE $y$ el aumento de dicha capacidad es de gran interés para los inversionistas no estatales. Para ampliar sobre la temática, véase la Ley General de Electricidad, nro. 17 812, publicado en La Gaceta nro. 170 el 1 de setiembre de 2010. Disponible en http://www.sise.co.cr/normativa/17-812. buscar, desde hace años, otros mercados en Centroamérica para colocar su producción (Arias, 13 de agosto de 2011).

La actitud de Arias evidencia que la presidenta Chinchilla falló en darle continuidad al proyecto energético concebido desde la administración pasada, máxime cuando la mandataria señalara que la propuesta del expresidente había sido modificada debido a que no tenía "viabilidad política", situación que molestó especialmente a Arias (Murillo, 11 de agosto de 2011). La modificación de las declaraciones, incluye ahora también una crítica a los grupos medioambientalistas $y$ a la prensa, por ser esta última opositora activa de las decisiones del gobierno en materia energética.

La principal crítica al Legislativo, radica en la creación de una comisión de diputados para discutir sobre la Ley General de Electricidad y su modificación posterior por un texto más sencillo. La alusión a este asunto es lo más recurrente de las noticias estudiadas para todo el periodo. Un aspecto importante es la pugna que existe dentro del Congreso con respecto a las propuestas sobre la ley energética. Los diputados del Partido Acción Ciudadana y el Frente Amplio no apoyaron a los oficialistas. Tal situación, según Arias ocasiona ingobernabilidad. En tal contexto, el diputado José Merino de Río entra en defensa de los grupos ambientalistas, acción que es atacada por La Nación, argumentando esta posición como un "discurso populista" y gastado.

A nivel general, la discusión política que enfrenta a los actores públicos con los privados se observa desde el 2006, cuando un grupo de productores privados acusa a la Asamblea Legislativa de no actuar para extender las licitaciones de las centrales eléctricas de capacidad limitada. Además, señalan que eso significa la obstrucción de las opciones para satisfacer la demanda ante la carestía de energía que se vive en ese año. René Castro, exministro de Ambiente y Energía durante el mandato de José Figueres Olsen, en uno de sus artículos de opinión en el año 2008, señala que "dentro de los inversores privados productores de biomasa está la familia del presidente Oscar Arias”. Asimismo, 
acusa al gobierno de desestimular la compra de energía a partir de dicha fuente por temor a que se diga que se podría beneficiar a esta familia, argumentando que se ha optado entonces por desarrollar plantas térmicas que emiten grandes cantidades de $\mathrm{CO}_{2}$ que contribuyen al calentamiento global (Castro, 27 de abril de 2008).

Igualmente, otros expertos argumentan a través de la sección de "Opinión" de $L a$ Nación, que a pesar de que es necesario desarrollar las fuentes renovables, no se debe desestimular la explotación de petróleo, pero esta debe estar liderada por el Estado y no por concesiones a empresas extranjeras (Roldán, 2 de agosto de 2011). Otros menos "moderados", como el exministro de Ambiente y Energía, Roberto Dobles ${ }^{13}$ y el empresario Ricardo Trujillo Molina $^{14}$, se declaran a favor de la explotación de gas natural.

Este último, por ejemplo, critica el alto costo de la producción de energía renovable y se pone a favor de la explotación del gas y el carbón, sin tomar en cuenta las críticas sobre los daños ambientales que ambos ocasionan y que son recurrentes en los medios. Menciona que este elevado costo sería trasladado a las facturas de

13 Roberto Dobles fue ministro de Ambiente y Energía durante el periodo presidencial de Óscar Arias entre 2006 y 2010.

14 El ingeniero Ricardo Trujillo Molina publicó cinco artículos de opinión en el diario La Nación durante el segundo semestre de 2011, todos argumentando en contra de las energías renovables y señalando las ventajas de la explotación de gas natural. los abonados y como solución plantea la apertura completa de la generación eléctrica, siendo el mercado el encargado de regular los precios por el servicio (Trujillo, 26 de agosto de 2011, 20 de octubre de 2011, 9 de noviembre de 2011, 24 de diciembre de 2011).

Por otro lado, se generan controversias en la prensa nacional con respecto a la implementación de políticas sobre energía renovable en el resto de países de América Central. Según los datos arrojados por el análisis de las notas que refieren a los diversos países de esta región, se observa un discurso casi generalizado que apunta a que en Costa Rica existe un retraso en materia de implementación de nuevas tecnologías de energía renovable, si se le compara con el resto de países.

Tal tesis es sostenida especialmente por los empresarios privados que se encuentran en pugna con el Poder Legislativo y que pretenden entrar a competir con el IcE. René Castro, incluso llega a realizar una comparación entre Costa Rica y Noruega, argumentando sobre el gran avance en materia energética del segundo y señalando que la generación en este país es 50-50 entre empresas estatales $y$ privadas. Finalmente, se concluye que tal situación no le ha generado ningún perjuicio al país europeo: "Hay que hacer que la concesión de obra pública funcione como debería y quitarse la idea de que todo lo privado es satánico", señala Castro refiriéndose al tema de la cogeneración eléctrica privada (Mesalles, 21 de marzo de 2009 y Castro, 11 de setiembre de 2011). 


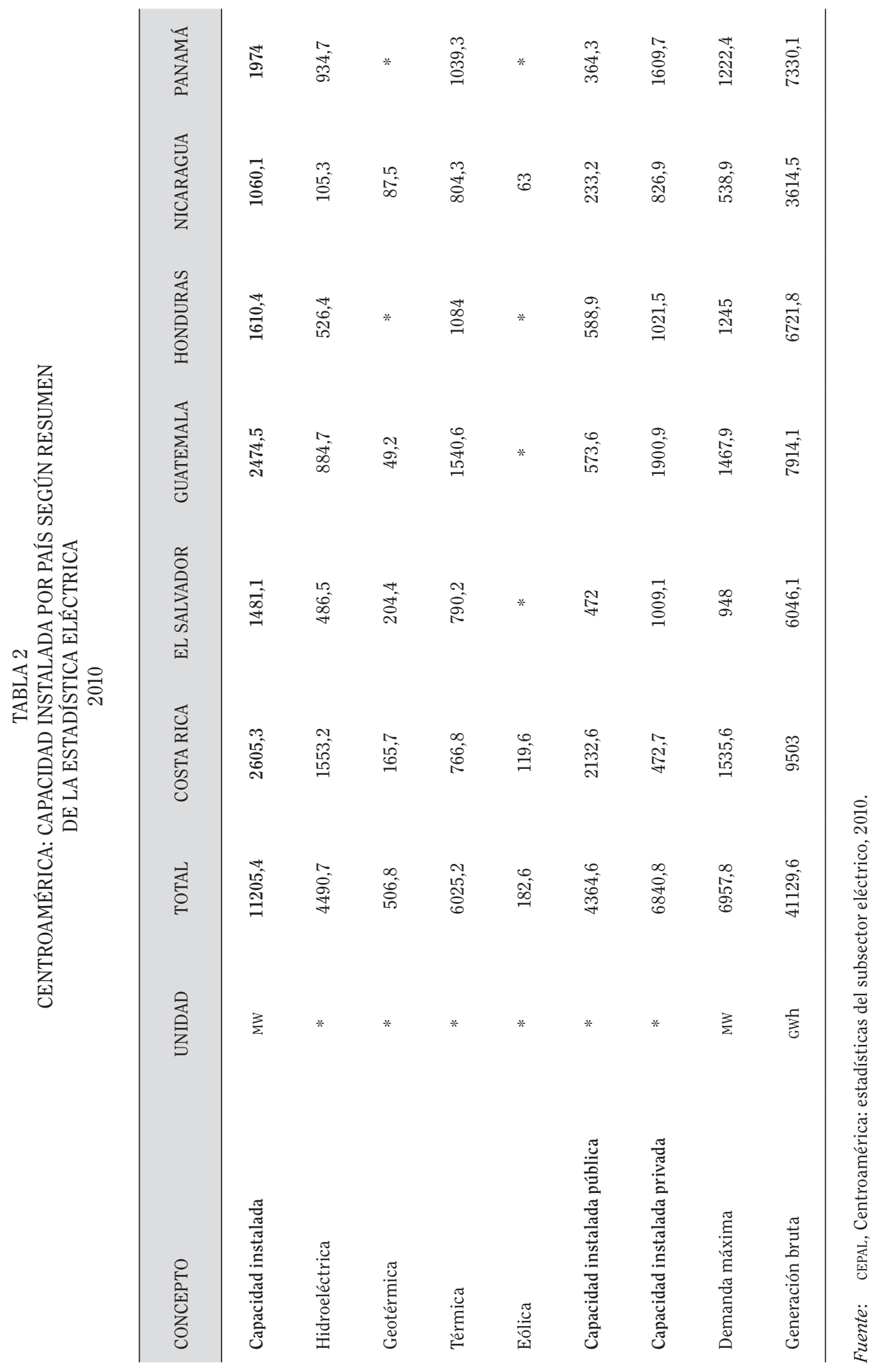


De la misma forma, el estudio de la CEPAL señala que las tarifas residenciales e industriales costarricenses son las más bajas de toda la región para ese periodo, contrarrestando la idea de los empresarios privados, quienes afirman que con el monopolio que mantiene el ICE, los precios se han disparado progresivamente. La idea de hacer de conocimiento público tales problemas, viene seguido de la auguración de que sus empresas resolverán ambos problemas, debido a que su producto es renovable. Por el contrario, se observa que en los demás países de la región, a pesar de que existe un predominio de la generación privada, la dependencia a los hidrocarburos es mucho mayor que para el caso de Costa Rica, lo cual se constata observando las cifras acerca de la capacidad instalada térmica, expuesta en la tabla 2.

Tal como se observa en la sección anterior, el periódico El Financiero concentra una gran cantidad de noticias sobre energías renovables en la sección Istmo (33\% del total). De la lectura conjunta de estos artículos, se pudo constatar que el medio de comunicación tiene un fuerte interés por mostrar los avances en la instalación de sistemas tecnológicos en la región, en contraposición al panorama adverso al que hay que enfrentarse en Costa Rica para poder establecer compañías energéticas. Es importante recordar que este medio es de carácter empresarial.

Lawrence Pratt, del Incae Business School, localizada en Costa Rica, señala por ejemplo, que el gran reto de los inversionistas centroamericanos en energía renovable es luchar contra las "trabas gubernamentales que impiden que este tipo de energía compita con las formas existentes", enfatizando en que las estructuras de los mercados actuales no son adecuadas para que las empresas privadas puedan insertarse (citado por Cordero, 2 de abril de 2008). Un punto interesante en este argumento, es que no considera el hecho de que la capacidad instalada de las empresas privadas es mucho mayor que la de las estatales en todo Centroamérica, por lo que el autor se estaría refiriendo a los inconvenientes que presenta la legislación en el caso específico de Costa Rica. Los medios, han señalado en diversas ocasiones, que los desarrolladores privados costarricenses han tenido que colocarse en otros mercados del istmo, por lo que se tienen claras las posibilidades regionales.

Igualmente, son importantes los actores privados como los internacionales. Para el caso específico de América Central, se observa que dichos países, en general, buscan fuentes de financiamiento externo para el desarrollo de proyectos de energía renovable. El actor estatal más importante en la región fue Brasil, con sus intentos por estimular la producción de etanol en la mayoría de países del área. En el año 2006, una delegación brasileña realizó una gira, empezando en Panamá y finalizando en Guatemala (La Nación, 26 de mayo de 2006). El objetivo de Brasil para este periodo fue legitimarse como una influencia regional en el campo de la producción energética, en contraposición a la Unión Europea.

Entre ambos actores internacionales existe una pugna por dicho mercado. Además, Brasil cuenta con la ventaja de que el $57 \%$ del etanol importado por Estados Unidos proviene de su país, de Costa Rica y El Salvador, lográndose introducir a ambos países en el mercado energético norteamericano. Pero en esta relación no solamente se negocia sobre la producción de energía renovable, como han indicado los sudamericanos: "el gobierno de Costa Rica ha invitado a la empresa estatal brasileña Petrobras a buscar petróleo en sus aguas" ( $L a$ Nación, 25 de abril de 2006).

De esta forma, el incentivo de exploración y explotación de fuentes no renovables no deja de estar presente en los discursos de la prensa, en los cuales se comparan los costos de la generación en Costa Rica con el resto de países centroamericanos, argumentando que en estos, los precios son mucho más bajos para el consumidor, debido principalmente a la explotación de los hidrocarburos. Trujillo (27 de junio de 2011) señala que las adversidades económicas nacionales con respecto a la generación energética se originan debido a que existe una negativa generalizada hacia la adopción de fuentes como el gas natural y el carbón. Este es uno de los principales expertos privados que afirma la condición rezagada de Costa Rica. 
Es importante introducir otros actores no estatales, como lo son los organismos económicos internacionales. Para el caso de América Central son principalmente el Banco Interamericano de Desarrollo (BID) y el Banco Centroamericano de Integración Económica (BCIE). El primero opta por invertir en el campo de los biocombustibles, sin embargo, está consciente de las fuertes críticas dirigidas a este tipo de energía, señalando que existe un "escepticismo de otros que afirmaban que los biocombustibles van a mover carros en los países ricos a expensas de la producción de alimentos en el mundo en desarrollo" (Ikeda, 2 de abril de 2007), refiriéndose a declaraciones como las que diera la Unión Europea y otras figuras regionales como Hugo Chávez y Fidel Castro, en contra de la "diplomacia de los combustibles" desarrollada por Brasil.

Estos últimos señalan, además de esta expansión del intercambio ecológicamente desigual con el Norte Global (Pérez-Rincón, 2007), que la producción de etanol es una empresa "promotora del hambre" (Renée, 10 de agosto de 2007). Por otra parte, el всіE con apoyo de la República Federal Alemana, llevaron a cabo el programa de "energías renovables y eficiencia energética" en Centroamérica, cuyo propósito es el de "contribuir a las iniciativas que impulsan la integración y el desarrollo energético regional" (El Financiero, 3 de marzo de 2006). En este sentido, Alemania tiene un gran peso, observado en varios de los artículos consultados.

Del estudio de los actores presentes en cada una de las noticias y de la interpretación de los datos numéricos procesados que se presentaron en la tabla 2 , se puede concluir que en los medios prevalece principalmente un confrontamiento ideológico entre los actores que tienen una visión más "desde el Estado" sobre la generación eléctrica y los defensores del mercado y la empresa privada, que representan la fracción oficialista del plenario y los inversionistas privados de origen nacional e internacional.

Se observa que los defensores del ICE $y$ de las políticas que amparan su funcionamiento, son los grupos de oposición al pLN y su escenario es principalmente la Asamblea Legislativa.
Entre ellos se destacan los diputados del Partido Acción Ciudadana (PAC) y Movimiento Libertario (мь), ambos en alianza, $y$ el diputado por el Frente Amplio José Merino del Río, quien fuera el más mencionado por la prensa de los actores en el Congreso. Debido más que todo a su crítica fuerte contra la privatización de entidades públicas y la reducción de la acción del Estado, así como al manifestarse a favor de las organizaciones ecológicas.

Asimismo, se encuentran los diputados del pun, sobre quienes recae el peso de las presiones ejercidas por el Ejecutivo para la aprobación rápida de las leyes referidas y entre quienes se genera una discrepancia interna debido al conflicto entre los mandatarios Arias y Chinchilla (Soto, 21 de agosto de 2011). Dentro del plenario, se percibe la negativa del pac en la introducción de la figura conocida como "gran consumidor", que son las grandes empresas consumidoras de energía que adquirirían el privilegio de negociar la compra con varios proveedores $^{15}$.

Dicho partido propone un proyecto nuevo que fue avalado por los diputados del Partido Alianza Sin Exclusión (PASE) y el Partido Unidad Social Cristiana (pusc). Su nuevo Plan de Contingencia eliminaría esta figura de gran consumidor, que atenta contra el principio solidario, además propone al ICE como único agente de mercado (agente monopólico, según principios económicos neoclásicos) y única entidad capaz de exportar energía. Además, aumenta la capacidad de generación de los sistemas privados hasta en un $45 \%$ del total nacional (Soto, 21 de agosto de 2011). Por último, el Frente Interno de Trabajadores del ICE (FIT-ICE), organizan manifestaciones públicas por la defensa de la institución y específicamente, contra los proyectos de ley promulgados por

15 En Costa Rica, las empresas transnacionales Intel, Cemex y Holcim son las únicas que superan el consumo de $10 \mathrm{Mw}$, por lo tanto, son las únicas que cuentan con este privilegio según la Ley 7200 . El proyecto de Laura Chinchilla, pretende entre otras cosas, bajar esta cifra a $1 \mathrm{mw}$, incluyendo al menos 75 empresas consumidoras más y que si se aprobara tal ley, estarían en la capacidad de negociar la energía con empresas privadas y ya no con el ICE, cooperativas o empresas de servicios municipales. 
ambas administraciones liberacionistas (Fumero, 26 de setiembre de 2011).

Por otro lado, se observa a los generadores privados y las nuevas empresas denominadas como "grandes consumidoras", quienes avalan el proyecto del Ejecutivo y que para defender su posición en los medios estudiados, señalan las deficiencias de los sistemas tecnológicos del ICE $y$ las ventajas competitivas de los demás países de América Central, exponiendo así las serias amenazas de "apagones" generales a partir de 2014. Además de remarcar las ventajas múltiples de las energías renovables, en relación con la reducción del consumo de petróleo, así como de las emisiones de GEI. El principal actor privado rastreado en las fuentes para este caso es la Unión Costarricense de Cámaras y Asociaciones del Sector Empresarial Privado (UCCAEP) junto con algunos expertos del INCAE, quienes se enfrentan en el ámbito público con el Poder Legislativo.

En los casos estudiados, también se observan organizaciones de la sociedad civil como los ambientalistas, quienes se manifiestan en contra de la explotación del petróleo y el carbón. Dichos actores son invisibilizados por los medios, a pesar de que cumplieran un rol fundamental en la moratoria que declarara el gobierno en el mes de agosto de 2011 contra la empresa transnacional Mallon Oil (moratoria hecha pública por René Castro recién nombrado como ministro), que adquirió una concesión en 1990 para explorar y explotar crudo en la zona norte del país. Igual que sucediera el año anterior, cuando se extendió una moratoria sobre la minería química a cielo abierto en la misma región del país, pero por parte de la empresa transnacional Infinito sA, controversia conocida como "Caso Crucitas".

La característica básica de estos actores para efectos de este estudio, es que obligaron a incluir una categoría para el análisis de las problemáticas que iba más allá del aspecto meramente económico y climático o la combinación de ambos. En este caso, se pudo identificar la existencia de controversias que se categorizaron como "conflictos de contenido ambiental", que como se vio anteriormente, se caracterizan por:
La existencia de una tensión entre intereses incompatibles con el medio ambiente de por medio [en donde] ninguno de los involucrados asume, necesariamente, la postura ética de defender el medio ambiente porque eso sea justo, noble o bueno. Lo que se reivindica es el bienestar material objetivo (o conveniente) de cada parte (Folchi, 2001, pp.12-14).

Esta característica según lo estudiado, sería aplicable también para el caso de las organizaciones locales de vecinos, que no defienden estrictamente la cuestión medioambiental, sino una afectación material de sus bienes privados. El caso más interesante que se identificó, corresponde a la manifestación frente a las instalaciones del proyecto hidroeléctrico $\mathrm{Pi}$ rrís, en donde un grupo de personas reclaman al gobierno de Laura Chinchilla, el día de la inauguración de la planta, por los daños ocasionados durante la construcción de esta, debido a que se socavó la tierra donde se encontraban sus cultivos y viviendas, así como, la generación de grietas en sus hogares. Posteriormente, la Comisión Nacional de Emergencias los obligó a abandonar sus bienes inmuebles y mover su domicilio. A esta manifestación se unieron otras comunidades que temen también por sus propios bienes.

\section{CONCLUSIONES}

Tal como se advierte a partir de estas múltiples controversias, los medios escritos analizados, combinan las construcciones subjetivas sobre el cambio climático y el calentamiento global con otros ámbitos de discusión no menos importantes, que involucran la capacidad de las estructuras de producción nacional, los recursos naturales disponibles, los patrones de producción y el consumo de energía. Asimismo, se observa que el tema de las implicaciones económicas de las políticas energéticas, tanto a nivel nacional como internacional, ocasionan una construcción de imaginarios sociales sobre las energías renovables, en donde convergen en el ámbito de discusión pública una serie de temas diversos, así como una variedad de actores que 
realizan una lectura asimétrica sobre el tema en cuestión (Viales, 2012).

Para estudiar lo anterior, se analizaron los principales discursos de estos actores en la construcción de dicho conocimiento por medio de la prensa. Este análisis se basó en las categorías temáticas definidas en la tabla 1 . En esta, se observa la cantidad de noticias que hacen referencia a los temas principales tratados, del total de 398 que integran el presente estudio. Igualmente, se utilizó la categoría correspondiente a los Actores clasificados por tipo, que se visibilizan por medio de La Nación y El Financiero, considerando a estos dos como los principales actores institucionales. Así, se observan las interacciones entre los actores privados, quienes son contemplados por el $58 \%$ del total de noticias; los públicos con un $44 \%$ y los internacionales con un $50 \%$ del total general de las fuentes.

La discusión presente en los medios se caracteriza por la existencia de un ímpetu político y económico, que argumenta a través de los medios de prensa escritos, la incorporación dentro del discurso energético nacional de nuevas fuentes de energía renovable, además de la tradicional energía hidroeléctrica desarrollada en Costa Rica, la cual ve amenazada su legitimidad mediática como alternativa a la dependencia del consumo de hidrocarburos. Así, la implementación de las TER en Costa Rica, se puede observar desde la óptica político-económica a partir de dos fenómenos específicos: 1) la crisis energética internacional y nacional, 2) el impacto positivo sobre el medio ambiente, como artefactos compensatorios a la emisión de dióxido de carbono (c02), que ha sido representada comúnmente por dichos medios como la principal causante del hipotético calentamiento global.

De esta forma, las energías renovables son proyectadas a futuro por dichos medios como las sucesoras de los hidrocarburos, por lo que fue de vital importancia explorar las iniciativas de los diversos actores privados por insertarse dentro de este lucrativo mercado, en el marco geopolítico planteado por el Protocolo de Kyoto, en el cual se propone como una de las soluciones al problema medioambiental, la transferencia de TER de los países más contaminantes hacia los países empobrecidos.
Además, se presenta una coyuntura que se caracteriza por las crisis energéticas que ocasionan el incremento en los precios del petróleo y el carbón, así como por los discursos nacionales e internacionales referentes a la hipótesis del calentamiento global, que viene a redefinir las políticas internacionales sobre la producción y el consumo energético (Viales, 2012). En Costa Rica, como se pudo observar, tal temática se inserta obligatoriamente en las controversias ocasionadas por el modelo eléctrico solidario gestado desde la década de 1950 $y$ en las supuestas incapacidades de generación de energía eléctrica por parte del ice.

\section{BIBLIOGRAFÍA}

\section{LIBROS}

Massarani, L. y Buys, B. (2008). Cuando la ciencia es noticia: una evaluación de la sección de ciencia en nueve países de América Latina. En Lozano, M. y Sánchez-Mora, C. (eds.). Evaluando la comunicación de la ciencia: una perspectiva latinoamericana. (115-130). México: CYTED, AECI, DGDC-UNAM.

\section{PUBLICACIONES PERIÓDICAS}

Alemania y BCIE impulsarán programa de energía renovable en Centroamérica (3 de marzo de 2006). El Financiero. Acceso en http://wvw.elfinancierocr. com/ef_archivo/2006/marzo/05/ lomasreciente643328.html

Arias, Ó. (13 de agosto de 2011). La ingobernabilidad y la seguridad jurídica. La Nación, Acceso en http://www.nacion. com/opinion/foros/ingobernabilidadyseguridad-juridica_0_1213478672.html

Castro, R. (27 de abril de 2008). Amenaza de shock eléctrico. La Nación, Acceso en http://www.nacion.com/opinion/ foros/Amenaza-emshock-emelectrico_0_9729028 23.html

Castro, R. (11 de setiembre de 2011). Ecodesarrollo. El Financiero. Acceso el 22 de noviembre de 2016. https://goo.gl/fbVmPB

Centroamérica presenta deficiencias en energía renovable (25 de abril de 2007). 
La Nación. Recuperado de: http://www. nacion.com/archivo/Centroamericapresenta-deficiencias-energiarenovable_0_826317370.html

Cerdas, G. (2007-2008). Agrocombustibles: las amenazas del imperialismo verde. Anuario de Estudios Centroamericanos, 33-34, 111-152.

Cordero, D. (2 de abril de 2008). Centroamérica ve negocio en energías renovables. $E l$ Financiero. Acceso en http://wvw. elfinancierocr.com/ef_archivo/2008/ abril/06/istmo1480530.html

Empresarios brasileños buscan socios estratégicos en Costa Rica (26 de mayo de 2006). La Nación. Acceso en http:// wvw.nacion.com/ln_ee/2006/mayo/26/ ultima-ce16.html

Folchi, M. (2001). Conflictos de contenido ambiental y ecologismo de los pobres: no siempre pobres, ni siempre ecologistas. Ecología Política 22, 79-100.

Fumero, J. (26 de setiembre de 2011). Un llamado a la innovación. La Nación. Acceso en http://www. nacion.com/archivo/llamadoinnovacion_0_1222277859.html

Ikeda, N. (2 de abril de 2007). BID anuncia inversiones de 300 millones para biocombustibles; ayuda a Centroamérica. La Nación. Acceso el 22 de noviembre de 2016. https://goo.gl/O3ozrk

Impulso al potencial eléctrico (19 de diciembre de 2009). La Nación. Acceso en http:// www.nacion.com/opinion/editorial/ Impulso-potencial-electrico_0_10930907 85.html

Ley General de Electricidad. (1 de setiembre, 2010). nro. 17.812. Publicado en La Gaceta No. 170. Acceso el 22 de noviembre de 2016. https://goo.gl/lj71HB

Mesalles, L. (21 de marzo de 2009). Letras de cambio. La Nación. Acceso el 22 de noviembre de 2016. https://goo.gl/3NS2ln

Murillo, Á. (11 de agosto de 2011). Óscar Arias insiste en que Costa Rica está perdiendo el tiempo. La Nación. Acceso en $h t t p: / / w w w . n a c i o n . c o m / n a c i o n a l /$ politica/Oscar-Arias-Costa-Ricaperdiendo_0_1213078827.html
Pérez-Rincón, M. A. (2007). El intercambio ecológicamente desigual del comercio internacional colombiano. Ecología Política, 33, (121), 121-123.

Renée, D. (10 de agosto de 2007). "Diplomacia de biocombustibles" de Lula no genera entusiasmo. La Nación. Acceso en http:// www.nacion.com/archivo/Diplomaciabi o c o mbustibles-Lula-generaentusiasmo_0_920707986.html

Roldán, C. (2 de agosto de 2011). Petróleo, gas natural o energías limpias. La Nación. Acceso en http://www.nacion.com/ opinion/foros/Petroleo-gas-naturalenergias-limpias_0_1211278895.html

Sarudiansky, M. (2016). Análisis temático sobre la fibromialgia en la prensa escrita argentina: descripciones generales y el rol de la psicología. Comunicação saúde educação, 20, (56), 25-36.

Soto Morales, E. (21 de agosto, 2011). Contingencia también se descarga. El Financiero. Acceso en http://wvw. elfinancierocr.com/ef_archivo/2011/ agosto/21/economia2871635.html

Takahashi, Bruno; Meisner, Mark (mayo de 2013). Climate change in Peruvian newspapers: the role of foreign voices in a context of vulnerability. Public

Understanding of Science, 22, 427-442. doi:10.1177/0963662511431204

Torre, T. de la. (28 de mayo de 2007). ICE no tiene dudas sobre futuro. La Nación. Acceso en http://www.nacion.com/ opinion/ICE-dudas-futuro_0_905909460. html

Trujillo, R. (27 de junio de 2011). Bajas tarifas eléctricas. La Nación. Acceso el 22 de noviembre de 2016. https://goo. $\mathrm{gl} / 5 \mathrm{hH} 5 \mathrm{u} 9$

Trujillo, R. (20 de octubre de 2011). Gas natural y recursos renovables. La Nación. Acceso en http://www.nacion.com/ opinion/foros/Gas-natural-recursosrenovables_0_1227077305.html

Trujillo, R. (9 de noviembre de 2011). Perdimos la ventaja competitiva eléctrica. La Nación. Acceso el 26 de agosto de 2011. http://www.nacion.com/opinion/ 
foros/Perdimos-ventaja-competitivaelectrica_0_1231076922.html

Trujillo, R. (26 de agosto de 2011). Tres opciones para el futuro del sector eléctrico. La Nación. Acceso el 26 de agosto de 2011. https://goo.gl/RKMfWk

Trujillo, R. (24 de diciembre de 2011). Una década de alzas en tarifas eléctricas. $L a$ Nación. Acceso en http://www.nacion. com/archivo/decada-alzasen-tarifaselectricas_0_1238676157.html

Un tope al desarrollo (12 de agosto, 2011). La Nación. Acceso en http://www. nacion.com/opinion/editorial/topedesarrollo_0_1213278720.html

OTROS

Blanco, J. M. y Fajardo, K. (2005). Informe final sobre Estrategia Energética Nacional. Undécimo informe sobre el estado de la nación en desarrollo humano sostenible. San José, Costa Rica.

Comisión Económica para América Latina y el Caribe, Centroamérica (2010).
Estadísticas del subsector eléctrico. Acceso en http://repositorio.cepal.org/ handle/11362/26072

Estudio General de Medios. IPSos-Media C.R. (2011 a 2012). Descripción general de La Nación. Acceso en http://www. nacionmediakit.com/contenido/ la-naci\%C3\%B3n.html

Procuradoría General de la República. (1995). Reforma a la Ley que Autoriza la Generación Eléctrica Autónoma o Paralela. Acceso el 22 de noviembre de 2016. https://goo.gl/KGyVRz

Viales, R. (2012). La Historia, el cambio climático y la hipótesis del calentamiento global. El cambio climático según el periódico "La Nación” (2009), Costa Rica. Una visión crítica. Trabajo presentado en las Jornadas de Investigación del Centro de Investigaciones Históricas de América Central, San José, Costa Rica.

Fecha de ingreso: 11/07/2016 Fecha de aprobación: 16/08/2016 
